\title{
Los incendios forestales y su impacto ambiental en las reservas ecológicas
}

\author{
Forest fires and their environmental impact on ecological reserves
}

\author{
Yolanda Tatiana Carrasco Ruano. ${ }^{1}$, María Soledad Núñez Moreno. ${ }^{2}$
}

\section{Resumen}

Existen varios tipos de incendios forestales, este trabajo detalla de manera específicamente los incendios que ocurren en los meses de verano. La mayoría de incidentes son ocasionados por ciudadanos que no tienen conciencia del daño causado al iniciar un incendio forestal, vulnerando los derechos de la naturaleza al no existir una cultura de conservación del medio ambiente, causando un grave impacto climático en el lugar donde se desarrolla la vida.

Objetivos: 1. análisis de las penas para este tipo de delitos, 2. análisis de las unidades especializadas en derecho ambiental para la aplicación de la normativa, 3. determinar el daño ambiental de las reservas ecológicas.

Método empleado: se examina críticamente la literatura legal y ecológica, para evaluar las respuestas a estos problemas, y poder exponer un enfoque propio al problema.

Aporte: es necesario mantener un desarrollo sostenible, es decir no contaminar el medio ambiente ya que la mayoría de incendios forestales han sido provocados por productos contaminantes y desechos, que combinados con el calor del verano inician los incendios.

Palabras claves: Impacto ambiental, incendios forestales, reservas ecológicas.

\section{Abstract.}

There are several types of forest fires, this work specifically details the fires that occur in the summer months. Most incidents are caused by citizens who are not aware of the damage caused by starting a forest fire, violating the rights of nature because there is no culture of environmental conservation, causing a serious climate impact in the place where the lifetime.

\footnotetext{
Visionario digital, Ambato, Ecuador, tatianacarrasco@cienciadigital.org

2 Escuela Superior Técnica de Chimborazo (ESPOCH), Riobamba, Ecuador, solenu0408@gmail.com
} 
Objectives: 1. analysis of the penalties for this type of crime, 2. analysis of the units specialized in environmental law for the application of the regulations, 3 . determine the environmental damage of the ecological reserves.

Method used: the legal and ecological literature is critically examined to evaluate the answers to these problems, and to be able to expose a proper approach to the problem.

Contribution: it is necessary to maintain a sustainable development, that is, not to pollute the environment since most forest fires have been caused by polluting products and wastes, which combined with the summer heat start the fires.

Keywords: Environmental impact, forest fires, ecological reserves.

\section{Introducción}

Según Guadalupe M. (2016), es importante trabajar para seguir subsistiendo en nuestro planeta, relacionándonos con el medio ambiente que nos rodea como seres vivientes de la tierra alrededor del mundo. Se hace concientización de lo peligroso que son quemas y tala de bosques pero al parecer debido a la gran sobrepoblación que hay casi es imposible evitarlo, en algunos lugares que aún se conservan reservas forestales que son las que contribuyen en gran mantener el clima un poco estable y toda la información requerida en este trabajo fue en su mayor parte adquirida a través de Internet la otra parte es de investigación que se hizo como grupo escalando en el que pudimos observar en realidad cual relacionado está en el escrito por medio de fotografías y esperamos que el final todos tengamos la dicha de vivir en este mundo disfrutando de la belleza natural y cuidar la vegetación y así contribuir a una prolongación de vida. Cuando los pajonales y bosques sufren los incendios forestales la pérdida de calidad paisajística es la consecuencia más fácilmente apreciable por la desaparición de la cubierta vegetal pero los incendios forestales son más destructivos y dañinos de lo que se puede observar a simple, afectan negativamente a todos los integrantes del ecosistemas estima que el 95\% de los incendios forestales tienen al ser humano y su actividad como causa de origen de hecho la actividad humana ha disparado la frecuencia de los incendios de modo que muchos ecosistemas sucumben de forma definitiva ante el poder devastador de las llamas altas temperatura que se generan en el interior de los incendios pueden llegar a eliminar los organismos encargados de desintegrar los materiales orgánicos, comprometiendo su fertilidad y la recolonización posterior por especies vegetales, diversas especies con menor movilidad son las que más se ven afectadas mientras que los demás animales probablemente escaparan del medio incendio y se refugiaran en zonas cercanas donde su presencia inevitablemente causara alteraciones en el equilibro ecológico de los procesos de deforestación son por lo general más destructivos en los trópicos la mayor parte de los suelos forestales son muchos menos fértiles de las regiones templadas y resultan fácilmente erosionables al proceso de lixiviación causado por la elevada pluviosidad que impide la acumulación de nutrientes en el suelo no obstante de las políticas coloniales se basaban en el supuesto de un bosque exuberante significaba suelos fértiles pretendían conquistar los bosques, sobre todo para destinarlos a los cultivos comerciales y la 
agricultura ya han dejado un legado de suelos exhaustos. Los incendios forestales originados por causas naturales o antropogénicas son considerados como uno de los factores de perturbación ecológica y de transformación del paisaje de los ecosistemas terrestres tanto en el Ecuador como el mundo influyen en la composición estructura funcionamiento y dinámica de los ecosistemas forestaciones en donde constituyen un cambio temporal o recambio de especies provocando por el fuego los incendios forman parte de la dinámica de los ecosistemas y son una herramienta ecológica de manejo varios autores ha indicado que muchos tasa vegetales se han adaptado a su presencia unas son resistentes, al mismo y otras incluso llegan a necesitarlos para su supervivencia y ya que ha sido afectado por incendios de gran magnitud desde hace décadas, sin embrago las interrogantes de como la incidencia reciente de incendios número y superficie afectada por años recientes y preferentemente en un periodo de varios años y cuáles son las tendencias probables hacia el futuro en destinos escenarios así como sus efectos son todavía incipientes de los incendios forestal es el fuego que se extiende sin control en terreno forestal o silvestre afectado a combustibles vegetales, flora y fauna un incendio se distingue de otro tipos de incendio por su amplia extensión, la velocidad con la que se puede extender desde lugar de origen su potencial para cambiar de dirección inesperadamente y su capacidad para superar obstáculos como carreteras ríos y cortafuegos que se extiende sin control en terreno forestal afectado a combustibles forestales situados en el monte para que la combustión ocurra hay tres elementos que deben estar presente además en la apropiada proporción (Gladis S., 2014 ).

\section{CRITERIO:}

Los bosques naturales debe apoyar la producción sustentable de una gran variedad de productos de la madera y otros preservar la capacidad del bosque para prestar servicios ambientales, la biodiversidad y proveer los medio de subsistencia para mucha gente incluyendo los moradores indígenas de los bosques o las tribus que representan patrimonios culturales en peligroso de extensión hay muchos tipos de bosques pueden proporcionar indefinidamente una fuente continua de madera y otros productos comerciales si son manejados correctamente al mantener un área bajo la cobertura del bosque se pueden controlar la erosión, se estabilizan las pendientes se moderan los caudales de los ríos se protegen los ambientes acuáticos se mantiene la fertilidad del suelo se preserva el habitad de la fauna y se obtienen productor forestales no igníferos que son importantes para las economía y hogares locales.

\section{Impacto Ambiental}

Los autores, Valle A. (2017) establece que la sostenibilidad es una tema que adquiere gran importancia cuando se habla del sector automotriz pues a medidas de la población va creciendo hay cierto incremento en su producción lo cual implica un mayor consumo de materias como vidrio, plástico, gasolina, acero combustible diésel u otro tipo de combustible generando impacto en el medio ambiente pero existen diferentes alternativas para reducirlo por ejemplo, la evolución que ha tenido la tecnología ha permitido la fabricación de vehículos más amigables con el medio ambiente y así darle mayor prioridad a su relación con el eterno y el consiente de su responsabilidad frente al entorno 
ambiental en sus actividades y siempre a la vanguardia de los avances tecnológicos requiere elaborar el Estudio de Impacto Ambiental de la Operación, sustentando en las depuradas técnicas de gestión ambiental que le permitan ejecutar sus actividades mantenido los altos estándares de calidad ecológica protegiendo los ecosistemas de su entorno y existen asuntos pendientes en materia ambiental. Uno de los más importantes es la deforestación que tiene una proyección de la batalla decisiva apenas empieza para indígenas y ecologías tras triunfo del sí en el referéndum ambientales esperan que se conforme una comisión técnica para definir donde se realizara la ampliación de la Zona del Yasuní creada para proteger a los pueblos aislados el fuego tiene varios uso que son tradicionales fundamentalmente en las áreas rurales como medio de ordenación para la preparación de tineras agrícolas para inducir la germinación de semillas para eliminar malezas y animales peligrosos en áreas de cultivo todo ello, según los estudios realizados tiene como resultado adicional el ahorro de combustibles maquinarias pesadas y ligeras esfuerzo humano, saneamiento del entorno y otros beneficios (Aura R., 2014).

Con frecuencia se sigue calificando como el medio más eficaz y económicamente factible de ordenación y de preparación de tierras para la agricultura sobre todo en circunstancias en que está vedado o limitado el uso de maquinaria herbicidas y otras técnicas para modificar los ecosistemas que exigen elevado consumo de energía fósil y humana para muchas especies de árboles el fuego es necesario para su reproducción ya que necesitan del calor para que conos se puedan abrir y sus semillas liberadas caigan al suelo y puedan germinar y por un lado sirven como sumideros de carbono lo que significa que tienen la capacidad de absorber el dióxido de carbono atmosférico a través del proceso de fotosíntesis, que extrae el carbono de la atmosfera y lo almacena en el suelo y los incendios, sequias, infestaciones de insectos y brotes de enfermedades como resultado del calentamiento causado por el hombre pueden poner en peligro muchos bosques y pastizales la desforestación es un gran problema en los trópicos donde vastas extensiones de bosques tropicales han sido talados y quemados. (Mongaba L.2018).

\section{CRITERIO:}

La importancia del fuego en los ecosistemas es un asunto complejo si bien como la sociedad fuimos educados a asociarlos en muchas ocasiones a destrucción y daño es lo cierto es que el fuego y los ecosistemas has establecido relaciones, donde incluso y algunos ecosistemas han desarrollado adaptaciones para depender de sus efectos como la reducción de competencia por malezas el saneamiento o control de enfermedades entre las plantas la liberación o incorporación de nutrientes y en algunos caso la germinación de algunas semillas y las relaciones del fuego con un ecosistema se definen en lo que se llama régimen del fuego que tiene que ver con la severidad, la intensidad, la escala especial, la estacionalidad y la fuente predominante de ignición es decir la presencia del fuego en un ecosistema especifico y atributos. 


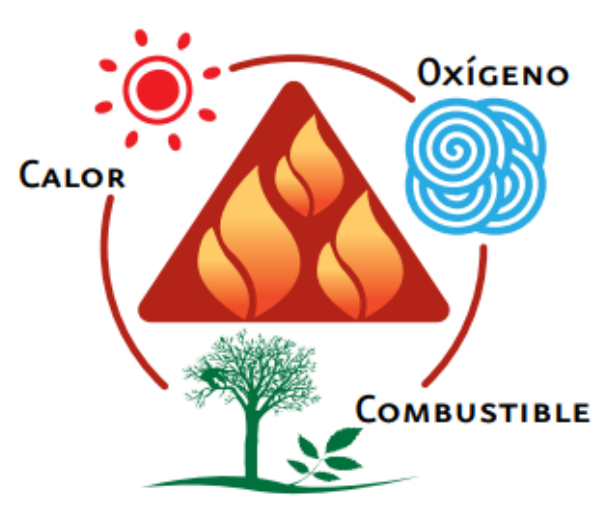

Figura 1. El fuego en el ecosistema

Autor: Elvis.A. (2014).

Fuente:http://www.conafor.gob.mx:8080/documentos/docs/10/236Gu\%C3\%AD a\%20pr\%C3\%Alctica\%20para\%20comunicadores\%20\%20Incendios\%20Forestales.pdf

Accidentales: Rupturas de líneas eléctricas, accidentes automolisticos, ferroviarios y aéreos.

Negligencias: Quemas agropecuarias no controladas fogatas de excursionistas, fumadores, que, de basura, limpieza de vas en carreteras y uso del fuego en otras actividades productivas dentro de áreas forestales.

Intencionales: Quemas por conflictos entre personas o comunidades tala ilegal o litigios.

Naturales: Caída de rayos y erupciones volcánicas

La fuente de calor: En el bosque no existe la combustión espontanea, siempre se requiere de una fuente de incandescencia externa mayor a $200 \mathrm{C}$ para que ocurra un incendio.

La temperatura: Los incendios forestales pueden ocurrir en cualquier momento, sin embargo, se presenta dos temporadas de mayor incidencia la primera, correspondiente a las zonas centro, norte, noreste, sur y sureste del país, que inicia en enero y concluye en junio. La segunda temporada inicia en mayo y termina en septiembre y se registra en el noreste de país ambas coinciden con la época de mayor estiaje en el territorio nacional.

Los asentamientos humanos: Una zona forestal a la que los humanos ingresan con facilidad y constancia es más susceptible a la ocurrencia de los incendios forestales.

\section{Los incendios forestales}

Los incendios es mayor de lo que se ya que en la mayoría de los casos sobrepasa los límites de las áreas afectadas la destrucción del flora y la fauna el aumento de la desertificación o la disminución de la calidad de las aguas y las atmosfera son algunas de las consecuencias negativas posteriores a un incendios y la recuperación de las zonas 
afectadas puede llevar décadas y los incendios modifican el agua generando perdidas de materia orgánica, cambiado la acidez de los ríos, lagos e impidiendo el desarrollo de la actividad ganadera además se dificulta y encarece la potabilización del agua ya que la misma arrastra cenizas y carbón por los mismo motivos el ciclo natural de agua se altera la recarga en el suelo es mucho menor el agua tiende más rápido generado mayor arrastre de sedimento los ríos ven sobrepasada su capacidad de transportar agua y se incrementan las posibilidades crecidas (Joha H., 20017).

\section{Medidas preventivas para evitar incendios forestales}

- No tirar colillas de cigarrillos encendida, ni fósforos

- No tirar colillas de desde vehículos en movimiento, sea en ruta o por caminos vecinales.

- Si no puede evitar fumar, preste atención a donde caen las cenizas, donde y como apaga el cigarrillo y lleves con usted la colilla.

- Si acampa hágalo en lugares autorizados, allí encender fuego

- Si observa un fogón encendido abandonado, apáguelo con abundante agua.

- Tenga cerca del fogón recipientes con agua.

- No arroje ni residuos en bosques y lugares de acampe, las latas y videos pueden actuar de lupa y provocar incendios.

- Prepare bien el fogón antes de empezar el fuego se debe colocar piedras alrededor de la fogata, hacer un pozo dentro para el fuego quede hundido en la tierra despejar de hojas y ramas alrededores de la fogata en lo posible amar una pared o proyección con piedras del lado de donde vives el viento.

- Nunca hay que hacer fuego de los árboles.

Los incendios forestación a escala mundial favorecen el efecto invernadero y el cambio climático a los niveles de dióxido de carbono en la atmosfera afectan gravemente a los suelos y generan condiciones que propician su erosión deterioran la calidad de las aguas y alteran los balances hídrico y sedimentario matan o producen graves daños a la vegetación modificando la composición, biodiversidad, estructura y madurez de la masas vegetales, modifica los hábitat y los recursos tróficos de los que depende la fauna cuta composición se verá afectada de manera selectiva alterando el equilibrio de los ecosistemas así como sus silos de materia y energía. (Néstor M. 2015).

Si los incendios forestales son uno de los principales problema ambientales, los espacios naturales protegidos son las áreas más importantes desde el punto vista de la conversación, porque contienen sistemas o elementos naturales representados, singulares, frágiles, amaneados o de especial interés ecológico, científico paisajístico, geológico o educativo, además de estar dedicados especialmente a la protección de la diversidad biológica de la geodiversidad y de los recurso naturales y culturales asociados. (Virginia C., 2016). 


\section{La Reserva Ecológica}

Las Reservas Ecológicas son áreas naturales terrestre o marinas de extensiones variables que pueden incluir uno o varios ecosistemas o formaciones vegetales en estado natural o con poca intervención humana revisten importancia nacional o regional para el manejo y utilización sostenible de los recurso naturales en beneficio de las comunidades humana ancestrales presentes al momento de su establecimiento es el objetivo principal es el de guardar materia genética, diversidad ecológica, bellezas escénicas, fenómenos especiales y la regulación ambiental para la investigación científica de elementos y fenómenos naturales y educación ambiental cuando no hay conflictos con la investigación y la educación se permiten actividades de recreación y ecoturismo en áreas limitadas siempre y cuando las características del recurso lo permitan (Moram, J. 2014).

El estado ecuatoriano ha mejorado el programa de protección de la naturaleza se han creado reservas ecológicas parque nacionales y áreas protegidas además de parques nacionales por otro lado se ha despertado en la ciudadanía el sentimiento de amor por el medio ambiente.

Áreas Reservas, Protegidas, Ecológicas y parque Nacionales de:

- Costa

- Sierra

- Oriente

- Galápagos

En la conservación de la diversidad biológica y la provisión de servicios ambientales, así como su potencial turístico por medio de la fortalezas naturales, turísticas y ambientales que existe en el lugar la mismo que sirvió como campo de batalla en la guerra con sus vecinos aún se observan en ella las trincheras utilizadas por las fuerzas armadas para la defensa del territorio nacional en la actitud es protagonista para la humanidad.

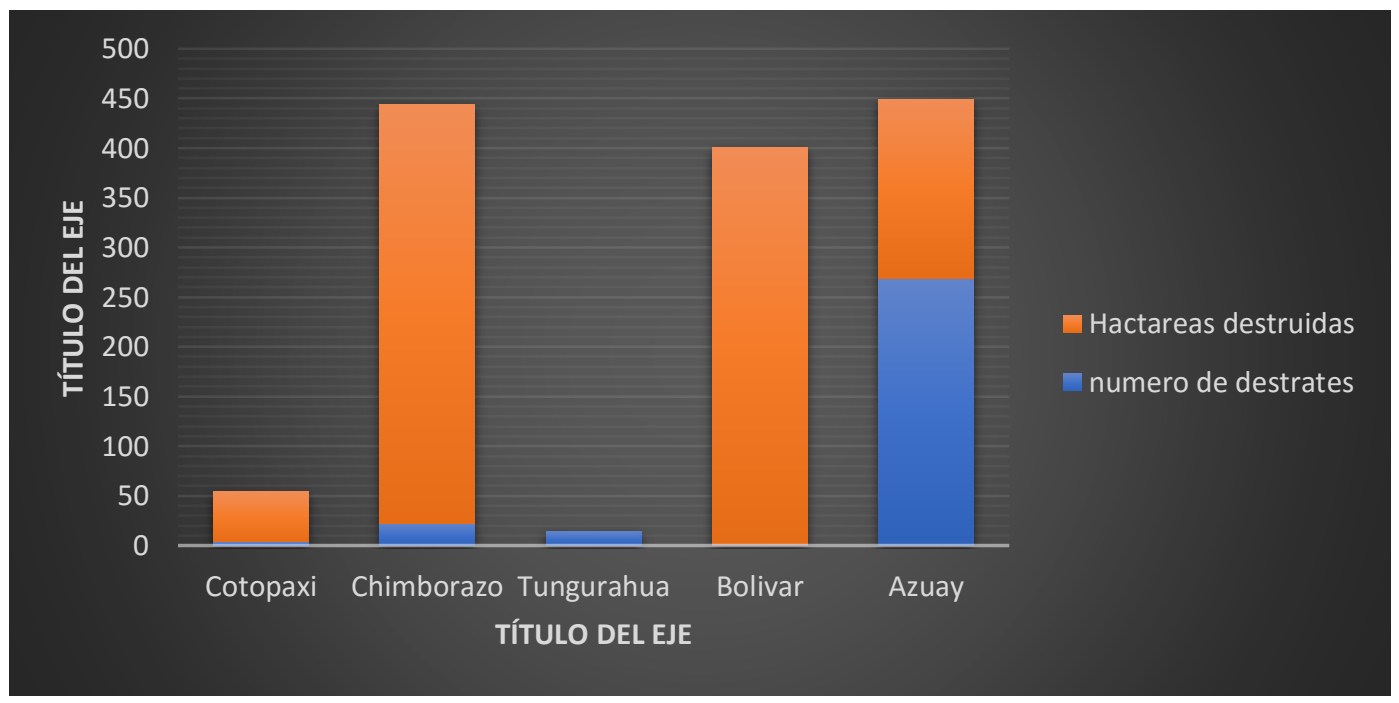

Figura 2: Los incendios de las provincias de Ecuador

Autor: Muñoz, G. (2016) 
Los incendios forestales de Ecuador mayores iguales a 2 hectáreas han ocasionado la pérdida de 480 de cobertura en 44 eventos, de estas provincias que reportaron un mayor números incendios fueron: Cotopaxi con 4, seguida Tungurahua con 14, seguida Chimborazo con 22. La provincia de la mayor afectación hasta el momento es Azuay con hectáreas quemadas seguida de Tungurahua, Chimborazo, Bolívar, Azuay y el Cotopaxi.

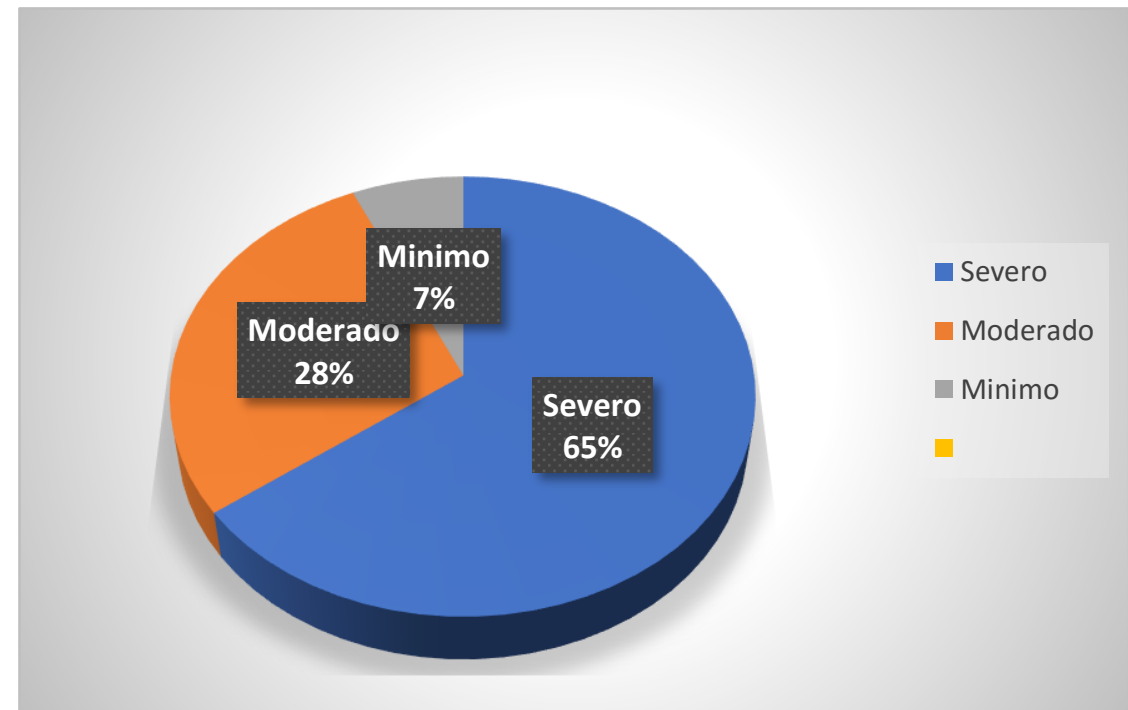

Figura 3: Incendios forestales de Ecuador

Autor: María, N. (2015)

Los incendios ocurridos en el país de 5.082 (64.87\%) este mínimo se espera una recuperación de área afectada de entre 1 y 2 años, 218 (28.38\%) de tipo Moderado a esta fue superficie le llevaras 5 años y solo 25 casos (6.75\%) fueron considerados de tipo Severo lo implica una recuperación al largo plazo más de 10 años, es que en los últimos años de incendios.

\section{Conclusiones}

- Los cambios ambientales que se dan la naturaleza son agentes negativos que alteran sus ciclos vitales en la actualidad se da por perdida una gran cantidad de áreas forestales, importantes para la purificación del aire y proteger el suelo.

- La valoración de estos daños ambientales se requiere del conocimiento de las condiciones previas y posteriores del impacto.

- La alteración negativa al estado medio natural ocasionado por acción de los incendios forestales constituye un daño falta al medio ambiente.

\section{Referencias Bibliográficas}

Aguilar.D. (26 de mayo de 2017). Obtenido de https://es.mongabay.com/2017/05/rafaelcorrea-ecuador-ambiente/ 
Diego.R. (2015). Los Incendios Forestales Vulneran los Derechos. Quito: http://www.dspace.uce.edu.ec/bitstream/25000/7391/1/T-UCE-0013-Ab347.pdf.

Elvis.A. (2014). Impacto del retroceso de los incendios. Quito: http://www.ambiente.gob.ec/wp-content/uploads/downloads/2014/07/PlanPreventivo-de-Quemas-Papallacta.pdf.

Gallegos.R. (2014). Investigaciones $\quad$ Forestales. México: http://www.redalyc.org/pdf/634/63439010010.pdf.

Gonzalo.C. (2015). Investigación de incendio de forestación. México: http://www.redalyc.org/pdf/634/63439010010.pdf.

Guadalupe.M.

(2016).

Desforestación.

Quito: https://www.monografias.com/trabajos14/deforestacion/deforestacion.shtml.

María.N. (2015). Impacto en la esfera forestal. Habana: http://ama.redciencia.cu/articulos/7.05.pdf.

Ñongaba.L. (10 de junio de 2018). Obtenido de https://es.mongabay.com/2018/06/ecuador-historias-ambientales-positivas2018/

Muñoz, G. (2016). Incendios desforestación. Quito: https://www.monografias.com/trabajos14/deforestacion/deforestacion.shtml.

Peralta Beltrán, A. (2015).

Peralta, B. (2015). Quito: http://www.indura.com.ec/Descargar/EIA\%20Expost\%20INDURA\%20Ecuad or $\% 20$ sucursal\%20Quito?path $=\% 2$ Fcontent $\% 2$ Fstorage $\% 2 F e c \% 2 F b i b l i o t e c a \%$ 2Fe70671ef6d6e48a9b7b3ee24ff664530.pdf. Obtenido de http://www.indura.com.ec/Descargar/EIA\%20Expost\%20INDURA\%20Ecuad or $\% 20$ sucursal\%20Quito?path $=\% 2$ Fcontent $\% 2$ Fstorage $\% 2 F e c \% 2 F b i b l i o t e c a \%$ 2Fe70671ef6d6e48a9b7b3ee24ff664530.pdf

Valle, A. (18 de agosto de 2017). Obtenido de https://://fierrosindustrial.com/noticias/impacto-social-ambiental-la-industriaautomotriz/https

Wright, P., \& Henson, B. (20 de abril de 2018). Obtenido de https://weather.com/science/environment/news/2018-04-18-earth-day-2018-10concerning-things-future-of-planet 
Para citar el artículo indexado.

Carrasco R., T., \& Núñez Moreno, M. (2019). Los incendios forestales y su impacto ambiental en reservas ecológicas. Explorador Digital, 3(3), 104-113. https://doi.org/10.33262/exploradordigital.v2i1.324

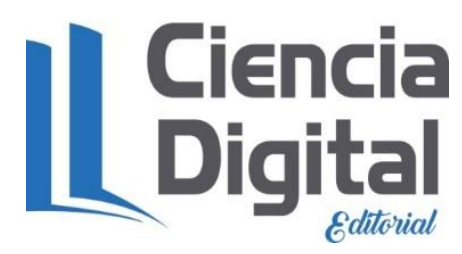

El artículo que se publica es de exclusiva responsabilidad de los autores y no necesariamente reflejan el pensamiento de la Revista Explorador Digital.

El articulo queda en propiedad de la revista y, por tanto, su publicación parcial y/o total en otro medio tiene que ser autorizado por el director o editor de la Revista Explorador Digital.

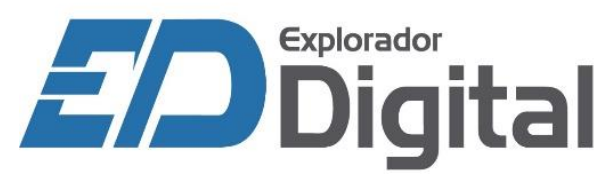

